\title{
DNA Repair Mechanisms in ESCs and iPSCs
}

\section{Pavan Rajanahalli*}

Department of Pathology, Immunology and Laboratory Medicine, University of Florida, Gainesville, Florida 32610, USA

Embryonic stem cells (ESCs) and induced pluripotent stem cells (iPSCs) have very stringent mechanisms of DNA repair compared to other somatic cells or terminally differentiated cells either by suppressing DNA damage or by elimination through apoptosis. ESCs are isolated from the inner cell mass of the blastocyst stage during embryonic development and iPSCs are reprogrammed from somatic cells that are formed using forced expression of stem cell master regulatory genes (Oct4, Nanog, Sox-2, c-Myc and/ Lin28, Klf-4) and exhibit very similar gene expression to ESCs. Both ESCs and iPSCs are pluripotent, have unlimited self- renewal capability and can differentiate into all 3 germ layers (ectoderm, endoderm and mesoderm) of the body [1]. To date, pluripotent stem cells can be differentiated into many cells types including neurons, microglia, cardiomyocytes, kidney cells, pancreatic $\beta$ cells, skeletal muscle cells, lung cells, liver cells and hematopoietic cells. Due to these properties, ESCs and iPSCs show promising outcomes in modelling diseases in a dish, regenerative medicine, drug discovery, personalised treatments and cell therapy [2].

Cell cycle and DNA replication in pluripotent stem cells (PSCs) occur at a faster rate (12-16 h) than somatic cells (24-36 h). DNA repair must be quick due to their short cell cycle phases and must be efficient to prevent further accumulation of mutations which could lead to tumor progression. PSCs have a very short G1 phase which prevents differentiation and increase cell proliferation which then maintains pluripotency. PSCs also exhibit a short G2 phase and most of the time is spent in the S phase which is in contrast to somatic cells [3]. Also, G1/S checkpoints controlled by retinoblastoma protein in PSCs are inactive, but active in somatic cells. This could further increase the mutational frequency in PSCs on subsequent cell divisions. With genotoxic and cytotoxic insults, if proper repair cannot be performed, cells undergo apoptosis in a very short time (around 5-6 h). An imbalance between pro and anti- apoptotic proteins leads to apoptosis of PSCs. Another option for PSCs with increased DNA damage is by differentiation thereby removing themselves from the cell population. PSCs spend most of their time in the S phase ( 70\%) [4]. Due to accelerated proliferation in PSCs, expression/functioning of cyclins in G2/M checkpoint is very different to somatic cells and can further increase the cell populations in the colony which harbour defective mutations in the $S$ phase. In terms of p53 regulating DNA damage in PSCs, due to the lack of G1 arrest and cell senescence, p53 independent apoptosis takes place after DSBs, but $\mathrm{p} 53$ deficient PSCs show high genomic instability which concludes that p53 is needed to maintain DNA stability and still be regarded as the guardian of the genome. Depending on the extent of DNA damage, low levels of DSBs can induce p53 to inhibit expression of Nanog and force the cells to differentiate (by down regulating Oct4, Sox2 and Nanog) thereby eliminating these PSCs from the main stem cell pool. Since p53 inhibits iPSC generation the levels of p53 are monitored carefully (by Sirt1 induced by Oct4) and is essential for maintaining pluripotency and surveilling DNA damage [5].

The common types of DNA repair mechanisms include Nucleotide excision repair (NER), homologous recombination (HR), nonhomologous end joining (NHEJ), base excision repair (BER) and mismatch repair (MR). Many of the stem cell master molecules including Oct4, Nanog, Sox-2, Sall4 are also involved in cell cycle regulation, DNA damage response, apoptosis and differentiation. In many cases if the damage is too severe, PSCs tend to undergo apoptosis through the mitochondrial pathway as cell senescence is not an option [6]. APRT/
HPRT when introduced into PSCs exhibit lower mutational frequencies than the differentiated cells suggesting mechanisms that are tightly regulating the PSC genomic stability. Supernumerary chromosomes are seen in abundance in PSCs during centrosomal amplification than in differentiated cells. Global gene expression analysis of PSCs have shown a high increase in DNA repair genes pertaining to double strand breaks (DSBs) and BER than somatic cells. PSCs express Zscan4 which helps in telomere elongation and HR. The most commonly used pathways to combat DNA damage are HR and NHEJ due to double strand breaks and ROS induced DNA instability is controlled by base excision repair and single stranded break repair. Also antioxidant genes are expressed at significantly higher levels than in somatic cells [7].

The various types of DNA repair mechanism employed depend on the stages of the cell cycle. HR repair occurs during the S/G2 phase of cell cycle with very high fidelity and HR related proteins stabilized by sall4 (RAD50, RAD51, RAD52, RAD54, MRE11 and NBS1) are elevated when damages are present during DNA replication. ATM is upregulated and hence amplification signals for DSB repair is enhanced by the phosphorylation of $\gamma \mathrm{H} 2 \mathrm{AX}$. During HR repair, the normal sister chromatid is readily available to serve as a template to repair the damaged chromosome/lesion and replace the mutated DNA with the normal sequence. However there is a possibility of loss of heterozygosity (duplication of the chromosomal segment or loss of heterozygous allele) that could be more serious than other DNA repair mechanisms $[8,9]$. HR is considered to be the primary repair mechanism in PSCs (to maintain proper pluripotency regulation and stem cell self-renewal) due to prolonged S/G2 phase. NHEJ repair predominates when DSBs occur due to radiations (UV and IR) and involves DNA ligation at the ends of the DSBs, but there can be possibilities of overhangs due to addition or deletion of nucleotides. NHEJ repair proteins (KU70 and KU80, XRCC4, LIG4) can be activated through all phases of the cell cycle as it does not require a normal template for repair, but most functional during early G1/S phases of cell cycle. NHEJ repair predominates during PSC differentiation. It has been shown that DSBs are more efficiently repaired with very few to no loss/addition of nucleotides during repair in PSCs, but higher number of mutational frequencies occurred in somatic cells $[9,10]$.

Nucleotide excision repair occurs when UV induced DNA damage occurs. Around 24-32 nucleotides are removed near the DNA lesion and DNA synthesis occurs followed by ligating the DNA. Global genome NER is significantly increased in PSCs than transcription coupled NER as seen in differentiated cells. Base excision repair is involved during removal of modified bases where DNA glycosylases

${ }^{*}$ Corresponding author: Pavan Rajanahalli, University of Florida, Department of Pathology, Immunology and Laboratory Medicine, 1275 Centre Dr, BMSB J522, Gainesville, Florida, 32610, USA, Tel: +1 (317) 407-7644, Fax: +1 (352) 273-9301, E-mail: pavan.raj@ufl.edu

Received October 02, 2016; Accepted October 04, 2016; Published October 11 2016

Citation: Rajanahalli P (2016) DNA Repair Mechanisms in ESCs and iPSCs. Mo Biol 5: e139. doi:10.4172/2168-9547.1000e139

Copyright: ( 2016 Rajanahalli P. This is an open-access article distributed under the terms of the Creative Commons Attribution License, which permits unrestricted use, distribution, and reproduction in any medium, provided the original author and source are credited. 
come into action to remove the modified nucleotide/s and form an abasic site. The empty site is filled in by the correct base and sealed covalently. $\mathrm{H}_{2} \mathrm{O}_{2}$ and 8-oxoguanine (regulated by OGG1) induced DNA damage is more efficiently repaired in PSCs due to high expression of BER genes than in their differentiated counterparts. Also, the kinetics of BER is shown to be faster in PSCs than in fibroblasts/differentiated cells $[11,12]$. PSCs also express high levels of ABC transporters that can efficiently efflux toxins (cytotoxic/genotoxic agents) from the cell. Mismatch repair (MMR) on the other hand can cause a mutator phenotype which can cause insertions, deletions and accumulation of mutations around DNA lesions (which can lead to abnormal replication fork formation) and can be detrimental to the cell. MMR proteins (MSH2, MSH6, MLH3, MLH1, PMS1 and PMS2) are elevated in PSCs and the efficiency of repair is at least increased by 5 fold in comparison to fibroblasts. It has been shown that iPSCs have higher copy number variations (CNVs) and chromosomal rearrangements compare to ESCs and fibroblasts. They may arise during or after the reprogramming process, or after clonal passaging or inherited from parental fibroblasts. Also New CNVs are formed as iPSCs proliferate in the culture making them unstable and susceptible to apoptosis. In iPSCs, the response to undergo apoptosis is reduced in comparison to ESCs which might be due to aberrant apoptotic signals. Responses to stressors in iPSCs can be different compared to ESCs and somatic cells [13]. Therefore DNA damage responses in iPSCs need to further be evaluated before they can be used in the clinic to avoid any complications of spontaneous mutations leading to tumor development, cell rejection, eliciting an immune response, toxicity or differentiation into unwanted cell types around the site of injection. DNA repair mechanisms in PSCs and somatic cells have different degrees of regulation at different stages of cell cycle. Since unrepaired DNA damage in ESCs and iPSCs can be propagated through development and affect all tissues of the body, repair mechanisms must be very accurate or PSCs undergo apoptosis. But in differentiated cells, there can be loss or gain of function if the mutation persists and affects only that cell population or tissue. It is also important to see if similar repair efficiency is maintained in iPSCs after reprogramming compared to ESCs since iPSCs are more sensitive to ionizing radiations. Genome wide association studies and whole genome analysis can reveal novel and complex mechanisms that govern genomic stability in iPSCs and ESCs [14].

In conclusion, DNA damage in PSCs mostly occurs through homologous recombination compared to NHEJ in somatic cells. High sensitivity to DNA damage, a low mutational burden, a higher antioxidant defence mechanism and very efficient DNA repair systems keep PSC populations free of DNA damage. PSCs undergo apoptosis if DNA damage is severe or un-repairable, but somatic cells try to repair the damaged DNA and do not undergo spontaneous apoptosis. HR predominates in PSCs having an extended S phase and high proliferation rate compared to somatic cells. Also telomere elongation factors are expressed at a higher level in PSCs, but are not activated in somatic cells which lead to telomere shortening. Glycolytic pathway is primarily used as a source of energy in PSCs compared to oxidative phosphorylation in somatic cells. Both ESCs and iPSCs have shown to have very less mitochondria compared to somatic cells. To reduce DNA damage insults in ESCs and iPSCs, culturing in hypoxic conditions, activating integrin signalling, using advanced growth medium, biocompatible $3 \mathrm{D}$ cultures which mimic cell growth and survival in vivo, reducing freeze thaw cycles, using low passage number of cells and constant feeding/monitoring can be helpful strategies that can be implemented for use in cell therapy.

\section{References}

1. Jinshui F, Carine R, Yoon-Young J, Hua L, Saul S, et al. (2011) Human induced pluripotent cells resemble embryonic stem cells demonstrating enhanced levels of DNA repair and efficacy of nonhomologous end-joining. Mutation Research 713: 8-17.

2. Martin DB, Lenhard RK, Tobias S (2015) Genome instability of ageing stem cells - Induction and defence mechanisms. Ageing Research Reviews 23: 29 36.

3. Yick WF, Carla I, Teppei Y, Claudia C, Ivan G, et al. (2011) A DNA repair complex functions as an Oct4/Sox2 co-activator in embryonic stem cells. Cell 147: 120-131.

4. Pavan R, Christopher JS, Yiling H (2015) The effects of silver nanoparticles on mouse embryonic stem cell self-renewal and proliferation. Toxicology Reports 2: $758-764$.

5. Pavan R, King DA, Meyer K, Zhu L, Wagner BD, et al. (2012) Conversion of mouse fibroblasts to sphere cells with differentiation potential induced by AlbuMAXI-containing medium. Frontiers in Bioscience (Elite Edition) 4: 18131822.

6. Elisia DT (2011) Mechanisms maintaining genomic integrity in embryonic stem cells and induced pluripotent stem cells. Exp Biol Med (Maywood) 236: 987996

7. Xuemei F, Ke C, Quuxiang Y, Lili Y, Yang X (2016) DNA repair mechanisms in embryonic stem cells. Cellular and Molecular Life Sciences, pp: 1-7.

8. Clarissa R, Reily R, Leticia KL, Oswaldo KO, Maria CM, et al. (2013) The role of DNA repair in the pluripotency and differentiation of human stem cells. Mutation Research/Reviews in Mutation Research 752: 25-35.

9. Kyle M, Pavan R, Maqusood A, John JR, Yiling H (2011) Toxicology in vitro 25 1721-1726.

10. Guido $F(2010)$ The bright and the dark sides of DNA repair in stem cells Journal of Biomedicine and Biotechnology 2010.

11. Luo LZ, Gopalakrishna PS, Nay SL, Park SW, Bates SE, et al. (2012) DNA repair in human pluripotent stem cells is distinct from that in non-pluripotent human cells. PLoS ONE 7: e30541.

12. Suvorova II, Katolikova NV, Pospelov VA (2012) New insights into cell cycle regulation and DNA damage response in embryonic stem cells. Int Rev Cell Mol Biol 299: 161-198.

13. Iyama T, Wilson DM (2013) DNA repair mechanisms in dividing and nondividing cells. DNA Repair 12: 620-636.

14. Prigione A, Fauler B, Lurz R, Lehrach H, Adjaye J (2010) The senescencerelated mitochondrial/oxidative stress pathway is repressed in human induced pluripotent stem cells. Stem Cells 28: 721-733. 